\title{
Anti-HER-2 Bispecific Antibody KN026
}

National Cancer Institute

\section{Source}

National Cancer Institute. Anti-HER-2 Bispecific Antibody KN026. NCI Thesaurus. Code C162577.

An eng ineered Fc-based heterodimeric bispecific monoclonal antibody, derived from trastuzumab and pertuzumab, directed against two distinct epitopes of the extracellular dimerization domain of the tumor-associated antigen (TAA) human tyrosine kinase receptor epidermal growth factor receptor 2 (HER2; ErbB2; HER-2), with potential immunomodulating and antineoplastic activities. Upon administration, anti-HER-2 bispecific antibody KN026 simultaneously targets and binds to two separate, nonoverlapping epitopes of HER-2, thereby inhibiting HER-2 heterodimerization and prevents the activation of HER-2 signaling pathways. By binding to HER-2, KN026 induces an antibody-dependent cell-mediated cytotoxicity (ADCC) ag ainst tumor cells that overexpress HER-2. This results in tumor cell apoptosis and inhibits tumor cell proliferation of HER-2-overexpressing tumor cells. HER-2, overexpressed on a variety of tumor cell types, plays an important role in proliferation, differentiation and survival. 